\title{
La Educación Comparada en los planes de estudio de ciencias de la Educación, en la universidad de Málaga
}

\author{
Ángela Caballero Cortés. \\ Facultad de Ciencias de la Educación. Universidad de Málaga. \\ acaballero@uma.es
}

\section{Resumen}

El objetivo de esta pequeña aportación, no es hacer un amplio recorrido histórico desde su nacimiento y desarrollo, hasta hoy día, de la «joven Universidad de Málaga». Pretendemos simplemente, poner de manifiesto, de forma breve, «algunos datos en sus 45 años de existencia. También dibujar a grandes rasgos, la historia del nacimiento en esta ciudad, de los Planes de estudios aprobados en la Dirección General de Enseñanza Universitaria, en la década de los años setenta y fechas posteriores, de las Titulaciones de Ciencias de la Educación (posteriormente Pedagogía) y (Psicología) durante el período que abarca de 1978 a 1983. En este recorrido histórico, analizaremos cómo surge, en los planes de estudio aprobados, la asignatura denominada Educación Comparada, en la Universidad de Málaga.

\section{Abstract}

The objective of this small contribution, is not to make a broad historical journey from its birth and development, to this day, the "young University of Málaga".

We simply want to show, briefly, some data in its 45 years of existence. Also draw in broad strokes, the history of birth in this city, of the Plans of studies approved in the General Direction of University Education, in the decade of the Seventies and later dates, of the Degrees of Sciences of the Education (later Pedagogy) and (Psychology) during the period from 1978 to 1983. In this historical journey, we will analyze how the subject called Comparative Education, in the University of Málaga, arises in the approved study plans.

Palabras clave: Málaga, Universidad, Ciencias de la Educación, Pedagogía, Educación Comparada.

Keywords: Málaga, University, Educational Sciences, Pedagogy, Comparative Education.

\section{HISTORIA E INTENTOS DE CREACIÓN DE LA INSTITUCIÓN UNIVERSITARIA MALACITANA}

El 18 de agosto de 1972 se promulgó el decreto por el que se creaba la Universidad de Málaga y fue publicado en el Boletín Oficial del Estado el 30 de septiembre, dotándola inicialmente de una Facultad de Ciencias Económicas y Empresariales y de una Facultad de Farmacia. Con el proyecto de Ley del III Plan de desarrollo la Universidad de Málaga, echó a andar con autonomía, con cuatro Facultades y tres Escuelas Superiores: Facultad de Económicas (dependiente de Granada), Medicina, Ciencias y Letras, la Escuela de Magisterio, Peritos y Comercio. Un reciente Colegio Universitario y una Facultad de Ciencias Económicas, constituían el único equipamiento universitario para una población cercana al millón de habitantes. Los tres centros históricos Superiores ya mencionados, ofrecían a los jóvenes malagueños la oportunidad de desarrollar sus capacidades intelectuales y conseguir una adecuada formación. 
En una publicación, titulada "Aniversario XXV: la Universidad de Málaga» (1998) ${ }^{1}$ se pone de relieve y se aborda la necesidad manifestada por la sociedad malagueña, el interés popular, la acción de las élites culturales, sociales, políticas, los medios de comunicación, así como la presión ejercida por la Asociación de Amigos de la Universidad de Málaga, para la creación de esta Institución, tan necesaria, demandada y argumentada, como era la creación de la Universidad de Málaga. Muchas eran las razones que, sobre su necesidad de creación, se daban: (Málaga séptima ciudad de España, en población de entonces, no contaba con un centro de estudios superiores» «única ciudad de Europa -se decía- con población superior a 300.000 habitantes, que no tiene Universidad»). Por tanto la creación de esta Institución era necesaria y urgente.

Varios han sido los escenarios del recorrido, físico e histórico, por los que han discurrido los centros de estudios, es decir el «conjunto de los estudios universitarios malacitanos»: El Colegio San Agustín, El Colegio Universitario de la Alameda, el Palo, Martiricos, El Ejido y Teatinos. Muchas son, hoy día, las Facultades, los edificios, los aularios, los laboratorios... que albergan las numerosas titulaciones, ampliamente diversificadas, que responden unas, a vocaciones personales y otras, a necesidades sociales. La concienciación de los malagueños a favor de una Universidad fue en aumento a medida que creció el número de habitantes y mejoraba su situación económica. Hasta los años sesenta del siglo XX, Málaga no se agrupa alrededor de un proyecto común : Su Universidad ${ }^{2}$.

Pasan los años y la ciudad de Málaga, por los años sesenta, con el apoyo de la prensa popular, entidades políticas y culturales, personajes destacados y los ciudadanos en general, está más volcada, en la consecución de centros de Enseñanza Superior. El primer paso hacia la Universidad se inicia con la creación de la Facultad de Ciencias Políticas, Económicas y Comerciales, en la ciudad. La alegría de la ciudad es lógica. Por todo ello no es de extrañar que en la tarde del 14 de mayo de 1963, un buen número de malagueños salga a la calle a expresar su alegría concentrándose en la Plaza de José Antonio. (Actual Plaza de la Constitución).

Fue la sociedad malagueña con sus movilizaciones y reivindicaciones, y la Asociación de Amigos de la Universidad las que lograron su universidad, ya que la vida académica en la ciudad dependía del distrito universitario de Granada, a donde tenían que viajar la mayoría de los jóvenes malagueños que podían, en esos años, acceder a los Estudios Superiores ${ }^{3}$.

\section{DATOS Y FECHAS DE INTERES}

En la citada publicación "Aniversario XXV: la Universidad de Málaga» se describe a Málaga, ciudad de rica tradición histórica y cultural. Fue fundada por los fenicios en el siglo VIII a.c y colonizada posteriormente por iberos, griegos, romanos árabes y cristianos. Su privilegiada situación geográfica, a orillas del mediterráneo y el carácter acogedor de sus habitantes, han hecho a lo largo de su historia, una ciudad moderna, cosmopolita y hospitalaria, que ha sabido encontrar su desarrollo en el comercio, la industria, el turismo y convertirse con su universidad, en un gran dinamizador social y cultural, así como ser el referente del nuevo soporte del futuro tecnológico, docente e investigador, hoy día.

En la joven historia de la universidad malacitana, destacar que en 1963 el Consejo de Ministros dispuso crear la Facultad de Ciencias Políticas, Económicas y Comerciales, dentro de la Universidad de Granada, pero con sede en Málaga.

\footnotetext{
${ }^{1}$ XXV años de la Universidad de Málaga (1998). Editado por la Universidad de Málaga.

${ }^{2}$ HIJANO DEL RIO, M. (1995). La Universidad de Málaga : Historia de una Institución docente en Educación y Cultura en la Málaga Contemporánea. Publicación Coordinada por M. VICO MONTEOLIVA que recoge una serie de investigaciones «Desde el sistema educativo Liberal (1785-1868) realizado por HIJANO DEL RÍO, M. «De las enseñanza profesionales a la creación de las Escuelas Técnicas (1785-1868) de GRANA GIL, I., «De la libertad de enseñanza a la creación del Ministerio de Instrucción Pública (1868-1900) de SANCHIDRIAN BLANCO, C., « Enseñanza Oficial y enseñanza Real (1900-1931) por MARTIN ZÚÑIGA, F., «La Educación en la Segunda República (1931-1937) de CABALLERO CORTÉS, A., Editorial Algazara y Universidad de Málaga . Málaga.

${ }^{3}$ Muchas son las publicaciones, en los distintos medios de comunicación: Diario Sur, La Opinión de Málaga, Málaga hoy... que, recogen los momentos de los inicios de los estudios universitarios en Málaga. En todas ellas « aparecen nombres como Francisco de la Torre y Cayetano Utrera, Luis Merino, Víctor Arroyo o Juan Peralta España... que recuerda Adelaida de la Calle, Rectora de la UMA, en ese año, en el discurso de inauguración de este curso académico así como en los actos de presentación del 40 aniversario de la UMA.
} 
El primer curso se inauguró el 10 de octubre de 1965. Esta primera Facultad funcionó en un edificio que acogerá después otras Facultades, convirtiéndose en un referente histórico : el número 23 de la Alameda Provincial. En esta historia, hay que contemplar la aparición, en la ciudad, de la Asociación de Amigos de la Universidad de Málaga, creada en 1968. Años más tarde las peticiones de los «malagueños» acompañadas por las realizadas por un centenar de procuradores en Cortes, hacen llegar al Gobierno la petición formal de cursos de Ciencias (Biología y Matemáticas) y Filosofía y Letras. El Colegio Universitario de Málaga, con el pronunciamiento favorable de la Universidad de Granada, comenzó a funcionar en el curso académico 1970/71. Al aprobarse los estatutos del Colegio- durante una reunión del Consejo de Rectores de la Junta Nacional de Universidades de España, celebrada en Málaga- se acordó dotarlo de Secciones de Ciencias, Letras, Medicina y Farmacia . El 5 de noviembre de 1971, cambió la historia de la ciudad de Málaga4. En la segunda mitad de los años 70 y la primera mitad de los 80, la Universidad de Málaga, fue desarrollándose fundamentalmente en su enclave inicial, el Ejido y con posterioridad en el nuevo Campus de Teatinos.

\section{ALUMNOSIAS MATRICULADOS EN SUS PRIMEROS AÑOS}

La Universidad de Málaga, en el curso 1972/73, tenía matriculados 2.230 alumnos en las Titulaciones de Económicas y Medicina. En el Colegio Universitario impartían docencia 203 profesores. Esta Universidad que contaba con unos 10.000 alumnos en el curso 1984/85, alcanzaba, trece años después, la mayor cantidad de alumnado: 40.000 , hombres y mujeres matriculados.

Grafico 1. Evolución de alumnos y alumnas matriculados en la universidad de Málaga.

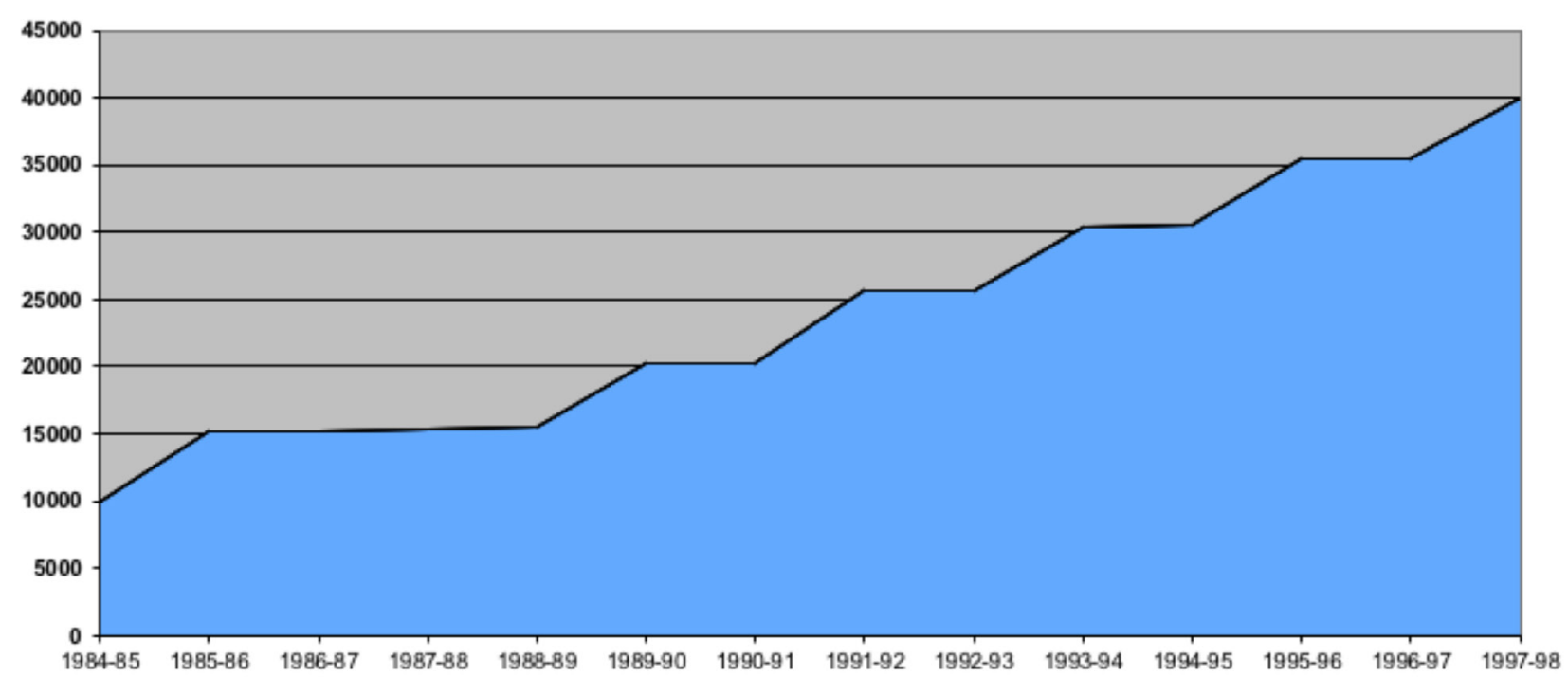

Los alumnos matriculados desde el curso académico 1991/92 hasta el curso 1997/98 comparten, por esos años, diferentes campos universitarios. Muchas de las Facultades que imparten dichas titulaciones se hallan ubicadas en el Ejido, otras se encuentran en el que será el campus de la Universidad malacitana en Teatinos. En número mayor de alumnos matriculados en estos años, provienen de la Facultad de Económicas, Filosofía y Letras, Derecho y Ciencias de la Educación.

\footnotetext{
${ }^{4}$ El Decreto de creación de la Universidad de Málaga se recibió con entusiasmo: Conocida la noticia, gente salió a manifestarse, pese a la lluvia. La prensa, las emisoras de radio, daban cuenta de la noticia. Al hecho se unieron la banda de música, las campanas de las iglesias de la capital y de la propia catedral.
} 
Grafico 3. Alumnos matriculados según rama de enseñanza y sexo.

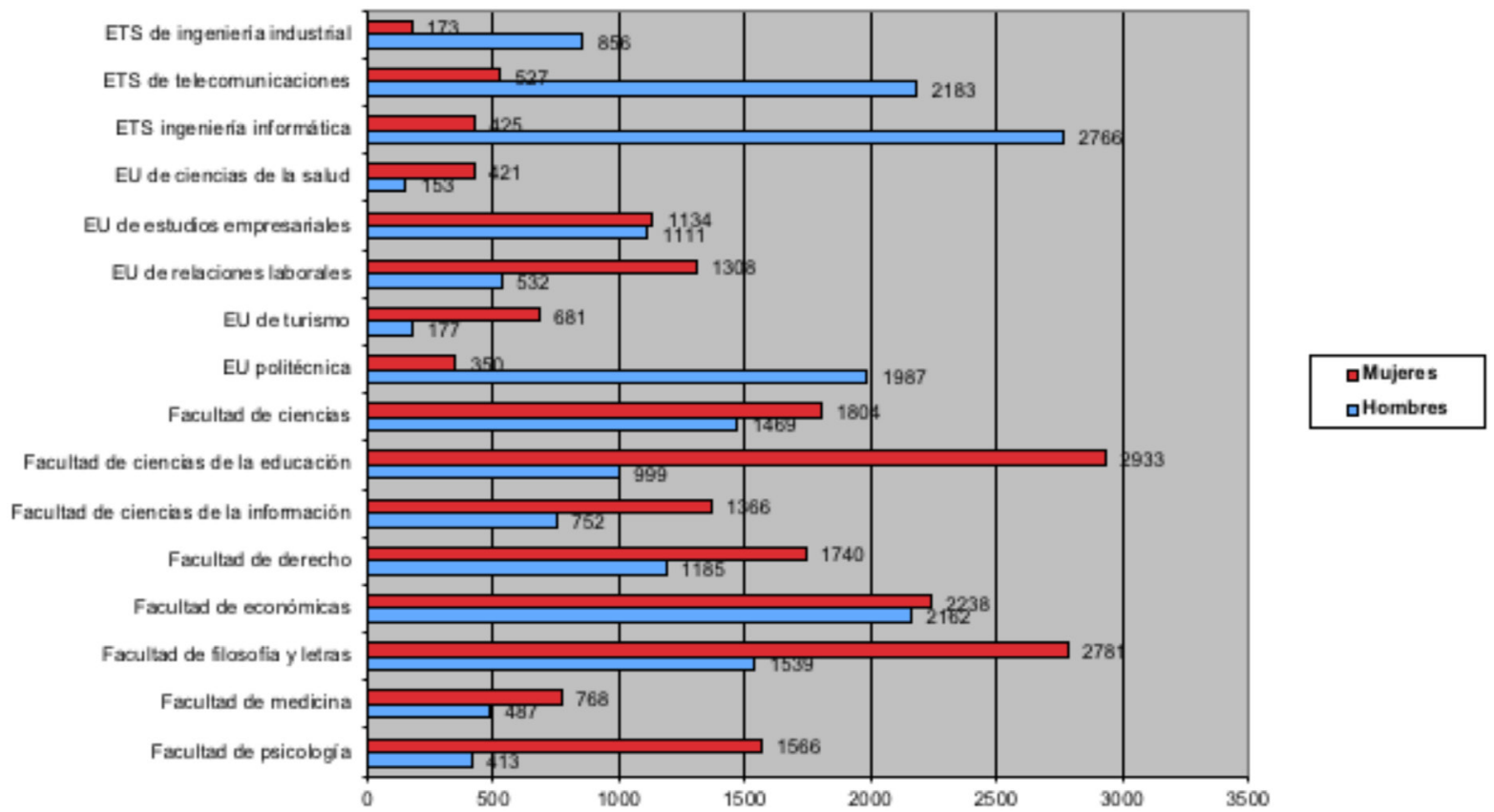

Fuente: Estadísticas de alumnos. Curso 2001-2002. Servicio Central de Informática. Universidad de Málaga.

\section{LA UNIVERSIDAD DE MALAGA HOY DIA. « LA UMA, 40 AÑOS COMPARTIENDO FUTURO»}

Con este lema, el día 11 de Octubre del 2012, en la presentación de los actos conmemorativos del 40 aniversario, en el Rectorado de la UMA, la rectora Adelaida de la Calle, destaca el salto cualitativo y cuantitativo dado en estos cuarenta años, cuatro décadas resumidas en las siguientes cifras: " de 2.300 alumnos en 1972 se ha pasado a cerca de 40.000 actuales, y de los 203 profesores a los 2.384 de ahora, de los que más de un $50 \%$ son doctores.

Los actos conmemorativos, han sido: 1. Inauguración en calle Larios de 25 paneles informativos de su evolución histórica. 2. Dar a conocer, en las dos salas del Edificio del Rectorado, la exposición «conoce tu Universidad». Los paneles expositivos hacen un repaso por la historia reciente de la Universidad, el ayer, el hoy, y el mañana de la UMA. 3. Presentación a la comunidad Universitaria y a la sociedad malagueña de los 40 años de historia.

\begin{tabular}{|c|c|c|c|}
\hline \multicolumn{4}{|c|}{ Tabla 1. Las últimas cifras de la Universidad en 2017 } \\
\hline & Alumnos matriculados & Hombres & Mujeres \\
\hline Grados & 38.218 & 14.178 & 16.040 \\
\hline Master Oficial & 2.576 & 1.151 & 1.425 \\
\hline Primer y Segundo ciclo P. Extinguir & 2.145 & 1.247 & 898 \\
\hline $\begin{array}{c}\text { Estudios de doctorado } \\
\text { Centros adscritos }\end{array}$ & $\begin{array}{c}1.122 \\
984\end{array}$ & \\
\hline
\end{tabular}

Fuente: La UMA en cifras. Dirección General de Comunicaciones e Información, 2015. 
En la actualidad, la Universidad de Málaga sigue apostando por el desarrollo científico y la innovación como vía para aportar progreso al conjunto de la sociedad. Destaca en estos años el fomento de la movilidad y el esfuerzo por atraer talento internacional, logrando una universidad abierta, cosmopolita y capaz de integrarse en proyectos científicos de máximo nivel: 45 años después, cuenta con más de 35.000 estudiantes, 58 títulos de Grado, 53 títulos de Máster, 278 grupos de investigación y 45 patentes registradas en 2011. Innovación, dinamismo e internacionalización son los principios que marcan la historia de la UMA y a su vez, la base establecida por la universidad para superar las dificultades actuales y reforzar su servicio por el conocimiento, por la sociedad y por el futuro.

\section{LA EDUCACION COMPARADA EN LOS PLANES DE ESTUDIO DE FILOSOFIA Y CIENCIAS DE LA EDUCACION. 1983}

En un excelente articulo titulado: «Prehistoria y primera historia de la Sociedad Española de Educación Comparada», sus autores, se aproximan a los orígenes de la Educación Comparada en España ${ }^{5}$. La publicación plantea los primeros vestigios de la Educación Comparada, la incipiente Institucionalización de la misma y los pasos iniciales de la Sociedad Española de Educación Comparada (1974-1994). Con un riguroso trabajo de investigación : «revisan el papel activo desarrollado por Juan Tusquets, que puso las bases para una primera institucionalización de la disciplina».

Fue en el marco del Plan de Estudio de 1969, de la Facultad de Filosofía y Letras, de la Universidad de Barcelona, cuando la Educación Comparada, adquirió carta de verdadera naturaleza académica. Juan Tusquets, acede a la presidencia del «núcleo germinal de la Sociedad Española de Pedagogía Comparada», en 1974. Le acompañan un grupo de profesores pertenecientes a tres universidades (Barcelona, Madrid y Valencia). A partir de 1977, accede a la presidencia el profesor Ricardo Marín Ibáñez.

Así surgen nueve centros de Educación Comparada: Barcelona, La Laguna, Madrid, Palma de MaIlorca, Pamplona, Salamanca, Santiago de Compostela, Tarragona y Valencia. Con posterioridad, se abren nuevos centros en diversas universidades: Murcia, Oviedo, Málaga y Granada.

A lo largo de estos años, se publican revistas especializadas, el Boletín de la Sociedad Española de Educación Comparada, y a partir de 1984, La Sociedad comienza a tomar conciencia de la necesidad de creación de su propia revista. Según los autores del artículo mencionado, el III Congreso Nacional de Educación Comparada celebrado en Málaga en 1987: lo definen «como un recuerdo que se mantiene vivo en cuantos participaron, en el mismo». Lamentablemente , a la hora de hacer balances, encontramos muchas personas-amigos-compañeros- que nos han dejado: Rosa Calatayud, Ricardo Marín, Ángel González, Florentino Sanz, Julio Ruiz Berrio, Ferrán Ferrer, Mercedes García de la Torre... Además de otros muchos, no sólo en el ámbito de la Educación Comparada, sino en otras áreas de conocimiento ${ }^{6}$. Sin el concurso de todos ellos, presentes y ausentes, no hubiera sido posible esta trayectoria de la disciplina, y de la Sociedad Española de Educación Comparada, afirman los compañeros del artículo mencionado. En la publicación del número 15, en la Revista Española de Educación Comparada ${ }^{7}$ los autores, «desarrollan los difíciles primeros pasos que tuvieron que dar aquellos que comenzaron el camino», allá por los años 60.

\footnotetext{
${ }^{5}$ Valls, R. Vilanou, C. Carreño, A. : Prehistoria y Primera Historia de la Sociedad Española de Educación Comparada. Revista Española de Educación Comparada, 24 (2014), 13-39. Los autores, en una rigurosa investigación, de personas, influencias, fechas...Nos muestran que existe una larga tradición en España con relación a la Educación Comparada que influyó de una y otra forma, en la génesis de la Sociedad Española de Pedagogía Comparada, nacida en 1974.

${ }^{6}$ RAMONA VLAS. IN MEMORIAN Joan Tusquets (1901-1998), Revista de Educación Comparada, 4 (1998), 287-289. J. L . García Garrido. IN MEMORIAN,: Ricardo Marín Ibáñez, Revista de Educación Comparada, 5 (1999), 293-294. y IN MEMORIAN,: Ángel González Hernández, Revista de Educación Comparada, 16 (2010), 11-12. Diego Sevilla. Instituciones y personas: La Sociedad Española de Educación Comparada 2002-2006. Revista de Educación Comparada, 24 (2014), 71-81. Luis Miguel Lázaro. IN MEMORIAN:Julio Ruiz Berrio y Ferrán Ferrer, Revista de Educación Comparada, 23 (2014), 9-12. En palabras de los compañeros, sobre Mercedes García de la Torre escriben « inicio su trabajo como docente en la Facultad de Educación de la Laguna en 1986 e impartió Educación Comparada desde dicha fecha hasta su retiro».

${ }^{7}$ Luis M. Naya, Ferrán Ferrer, M. Jesús Martínez Usarralde (2009). Pasado, presente y futuro de la Enseñanza de la Educación Comparada en España, 15, 277-294.
} 
Tomando las palabras de los autores: «los nombres de Juan Tusquets y Ricardo Marín pasaban a formar parte de un pasado doctrinal y societario, habiendo sido renovados por José Luis García Garrido, y un grupo de personas, entre ellos: Miguel Ángel Pereyra, Francés Raventós, Mercedes Vico, Ángela Caballero, Ángela del Valle, Vicente Llorent, Diego Sevilla, Clementina García, Leoncio Vega, Francisco Canes, Luis María Naya, Inmaculada Egido, que con ilusión y capacidad desde sus respectivas Universidades, han acentuado los vasos comunicantes con el exterior, sin olvidar los aspectos y problemáticas interiores». Desde mi recuerdo, quiero agregar, al listado de compañeros mencionados, algunos nombres más, y seguro que faltan algunos: Isabel Cors, Amalia Ayala, Antonio Luzón, Mónica Torres, Javier Valle, Gabriela Osenbac, Elisa Gavári, María José García, Teresa Terrón, María Jesús Martínez, Juan María Senent, Inmaculada González, María Rosa Oria...

En este ámbito de la Educación Comparada, hay que mencionar también, el papel del profesor José Luis García Garrido. En un artículo publicado en la Revista de Educación Comparada, él mismo enumera y le da el protagonismo a muchos compañeros que han escrito «a mi modo de ver la historia», no sólo de la Revista, de la Sociedad, de los boletines, de la disciplina de Educación Comparada, sino de la amistad y el compañerismo, que ha existido y lo sigue haciendo, de todos/ as aquellas personas, unos presentes, algunos desgraciadamente ausentes, que a lo largo de estos años, han compuesto las distintas Juntas Directivas, y han potenciado una serie de valores, personales y académicos. Entre todos/as hemos conseguido potenciar nuestras disciplinas académicas, estar presentes en congresos Nacionales, Internacionales y Mundiales. Aportar investigaciones y darlas a conocer, en la hoy prestigiosa Revista de Educación Comparada... en una palabra» sentirse una familia de comparatista en un contexto Internacional ${ }^{8}$ ». José Luis, al escribir el artículo, «hace memoria de los 20 años atrás, en la UNED, de una treintena de colegas, presididos y alentados por Ricardo Marín Ibáñez, decididos todos a dar un giro importante a la gestión de la que hasta entonces había sido nuestra Sociedad Española de Pedagogía Comparada (SEPC)». El artículo « escrito en primera persona y de modo casi autobiográfico, desea reflejar cuales fueron las razones del cambio y sobre todo la vida de la renovada asociación durante los primeros años, correspondiente al periodo de presidencia de su autor». En un apartado del mismo, habla de los protagonistas del cambio: Ricardo Marín, Julio Ruiz Berrio, Ángel González Hernández, Florentino Sainz Fernández y Ferrán Ferrer Juliá. "personas fallecidas, a las que la SEEC debe tanto y a las que me parecía de rigor dedicar ahora este sucinto homenaje». La Junta Directiva que se constituye en la UNED, antes aludida estaba compuesta, como Presidente: José Luis García Garrido, Vocales: Ferrán Ferrer, Ángel González, Ángela Caballero, Luis Miguel Lázaro9.

\section{LOS ESTUDIOS DE PEDAGOGÍA EN LA UNIVERSIDAD DE MALÁGA, EN SUS 45 AÑOS DE HISTORIA (1972-2017)}

Aunque los intentos de crear « estudios universitarios en Málaga» se remontan al Siglo XVI, hasta el curso 1972/73 no se produjo la integración de los Centros Universitarios de Málaga, adscritos a la Universidad de Granada, en la nueva institución malagueña. Referente a los Estudios en Ciencias Sociales, García Maldonado en su publicación sobre La Asociación de Amigos de la Universidad de Málaga, «documento vivo del sentir del conjunto de la sociedad malacitana, argumenta, razona y justifica, por primera vez, la necesidad de establecer en esta ciudad los estudios de Pedagogía y Psicología». Sobre Pedagogía: "Sería muy conveniente en orden al problema docente general de toda la provincia de Málaga, y como cantera del personal dedicado a la enseñanza. La provincia de Málaga tiene 21.890 niños y niñas en edad escolar, sin escolarizar ${ }^{10}$ ».

\footnotetext{
8 J. L. García Garrido. Los primeros años de la Reconstituida SEEC. Revista de Educación Comparada, 24 (2014), $41-54$.

${ }^{9}$ Palabra dJosé Luis: « La Universidad de Málaga, que había tenido en la anterior Junta de la SEPC, una representante de excepción (la profesora Mercedes Vico), siguió teniendo una presencia determinante en la vida de la renovada Sociedad.

${ }^{10}$ GARCIAMALDONADO,A.(1993).LaAsociación deAmigos delaUniversidad de Málaga. Ellogrodeunanhelodesiglos. LiceodeMálaga. Málaga. (NOTA) Comentarios de la Autora: La propiedad de este edificio, Padres Agustinos y las condiciones físicas del mismo, conllevan al traslado del profesorado y alumnado matriculado en el mismo, a otro edificio en el centro de la ciudad conocido como Colegio Universitario, sito en la Alameda. Este edificio propiedad del Ayuntamiento de Málaga, tiene un uso, multifuncional .Por la mañana sirve como archivo municipal, ya que es donde están depositados fondos documentales de la ciudad, y varios servicios municipales. Por la tarde, junto al desarrollo de la docencia, coincide con otras actividades organizadas por el Ayuntamiento, como «es el ensayo de la banda municipal».
} 
En el Edificio de la Alameda se iniciaron los cursos de Filosofía y Letras. Estos estudios pasaron mas tarde al también histórico Colegio de San Agustín, donde estuvo hasta 1985, cuando se instaló definitivamente en el Campus de Teatinos. En Málaga en el curso académico 1979-80, dependiendo de Filosofía y Letras, empiezan a impartirse en el centro de la ciudad, edificio de San Agustín, unos «estudios conducentes a la obtención de unos títulos, no muy definidos en sus comienzos...» $\left.{ }^{*}\right)$

Es el Departamento de Filosofía de la Facultad de Filosofía y Letras el encargado de organizar los tres primeros cursos académicos, confeccionando e impartiendo: « un Plan de Estudios compuesto por una serie de asignaturas destinadas a tal fin». Estas enseñanzas son impartidas por el profesorado adscrito a diferentes áreas de conocimiento.

En este apartado quiero hacer mención al Profesor José Manuel Esteve Zarazaga, que en mi modesta opinión, «organizó los estudios, que conducirían con posterioridad a las distintas promociones de ese Plan de Estudios a licenciarse, en estos estudios universitarios ${ }^{11} »$. También mencionar a la Profesora Mercedes Vico Monteoliva, que se incorpora a la Universidad de Málaga en 1983. Se encargó de dirigir el Departamento denominado Pedagogía Sistemática, donde estaban ubicadas las distintas asignaturas, que en aquellos años, componían los estudios conducentes tanto a estudios de Ciencias de la Educación como a Psicología ${ }^{12}$. En este primer cuadro aparecen las asignaturas impartidas desde a (1978-1983).

\footnotetext{
11 Julio Vera: José Manuel Esteve: sus contribuciones al estudio de la profesión docente. Revista Española de Educación Comparada, 22, (2013) 207-223. José Manuel Esteve (1951-2010). En 1980, llega a la Universidad de Málaga como profesor adjunto numerario «puso en marcha la Sección de Ciencias de la Educación embrión de la actual Facultad de Educación.».

${ }^{12}$ NOTA. Casualidades de la vida, académica y profesional, hablando en primera persona, tengo que decir, que yo fui alumna de esa primara promoción. Tuvimos como profesor en dos asignaturas, al organizador de los estudios de ese plan de estudio, a José Manuel Esteve Zarazaga, tristemente fallecido en 2010 y recordado con mucho cariño. No tuvimos, como he comentado anteriormente, en el plan de estudios cursado, la disciplina: Educación Comparada. Esta asignatura aparece con posterioridad en el siguiente plan de estudio, como optativa en quinto curso. En 1984, de forma casual, conozco a la Profesora Mercedes Vico Monteoliva, que llegó a Málaga, como Catedrática de Historia de la Educación, en 1983. Me habla de hacer la Tesina y con posterioridad la Tesis Doctoral. En el curso 1985/86, concurso y obtengo, una plaza de ayudante, en el Departamento de Teoría e Historia de la Educación, y aquí sigo hoy día. He sido profesora ayudante 1985/87. Profesora Asociada 1987/91. Profesora Titular interina 1991/93 y Profesora titular 1993/ hasta hoy. La Tesis Doctoral, siguiendo una de las líneas de investigación iniciada en el Departamento, sobre la historia de la Educación en Málaga: La Enseñanza Primaria en Málaga 1931-1951. En la distribución de la Docencia para los siguientes cursos académicos, 1986// 89, soy la encargada de impartir la asignatura Educación Comparada, optativa en quinto curso de carrera, junto a la Historia de las Instituciones Docentes, optativa también, y una asignatura troncal: Filosofía de la Educación.
} 
El origen de este Plan de Estudios está aprobado por la orden de 19 de junio de 1975 (B.O.E. de 29 de julio) y de 1 de Octubre de 1976 (B.O.E. de 2 de agosto de 1977). Plan de estudios cursados por la primera promoción de estos estudios.

\begin{tabular}{|c|c|c|c|}
\hline & ASIGNATURAS & & ASIGNATURAS \\
\hline & Historia de la Filosofía Medieval & & Historia de la Educación II \\
\hline PRIMER CURSO & Lógica & CUARTO CURSO & $\begin{array}{c}\text { Organización y Legislación } \\
\text { Escolar }\end{array}$ \\
\hline \multirow[t]{5}{*}{ CURSO 1978-79 } & Lengua Española & CURSO 1981-82 & Orientación Educativa II \\
\hline & Antropología & & Didáctica II \\
\hline & Psicología General & & Diseños Experimentales \\
\hline & & & Pedagogía Diferencial \\
\hline & Historia de la Filosofía Moderna & & \\
\hline SEGUNDO CURSO & Ética & & \\
\hline \multirow[t]{6}{*}{ CURSO 1979-80 } & Historia de la Psicología & & Psicología de la Educación \\
\hline & $\begin{array}{l}\text { Fundamentos Biológicos de la } \\
\text { Personalidad }\end{array}$ & QUINTO CURSO & Pedagogía Experimental \\
\hline & Psicología Evolutiva & CURSO 1982-83 & Didáctica Especial \\
\hline & Didáctica & & Educación Especial \\
\hline & & & Orientación Educativa \\
\hline & $\begin{array}{c}\text { Historia de la Filosofía } \\
\text { Contemporánea }\end{array}$ & & Tecnología de la Educación \\
\hline TERCER CURSO & Estadística & & \\
\hline \multirow[t]{6}{*}{ CURSO 1980-81 } & Historia de la Pedagogía & & \\
\hline & Teoría de la Educación & & \\
\hline & Psicodiágnóstico & & \\
\hline & Psicometría & & \\
\hline & Ética & & \\
\hline & Didáctica & & \\
\hline
\end{tabular}

Fuente: Ángela Caballero Cortés. Elaboración propia como representante de la Primera promoción de estos estudios. Información obtenida de su Certificación Académica Personal y de las «papeletas acreditativas de las asignaturas cursadas», así como los nombres del profesorado encargado de impartir la docencia de cada una de las asignaturas.

\section{EL CENTRO DE PEDAGOGÍA COMPARADA EN MALAGA (1984-1994)}

En este breve apartado en los diez años de historia del centro en Málaga, fue el encuentro con Mercedes Vico, con sus propuestas académicas, su interés por conocer e integrarse en la Málaga de acogida, el relato de sus proyectos, entre ellos la creación de un Centro de Pedagogía Comparada, la que hizo realidad que en esta ciudad, un grupo de personas, que ya formaban parte del Departamento, con otro grupo de personas procedentes de la enseñanza publica, concertada, inspección, alumnado... se unieran para hacer realidad la creación el centro mencionado. El 20 de enero de 1984, en la Unidad Docente de Educación Comparada e Historia de la Educación del Departamento de Pe- 
dagogía Sistemática de la Universidad de Málaga « leídos los estatutos de la Sociedad Española de Pedagogía Comparada, y estando de acuerdo con los mismos se decide: elevar escrito al IImo. Sr. Presidente de la Sociedad Española de Pedagogía Comparada, proponiendo la creación del CENTRO DE PEDAGOGÍA COMPARADA DE MALAGA». En el acta de constitución, se expone para su aprobación-si procede, en la Asamblea que se celebrara el día 28 de enero de $1984^{13}$.
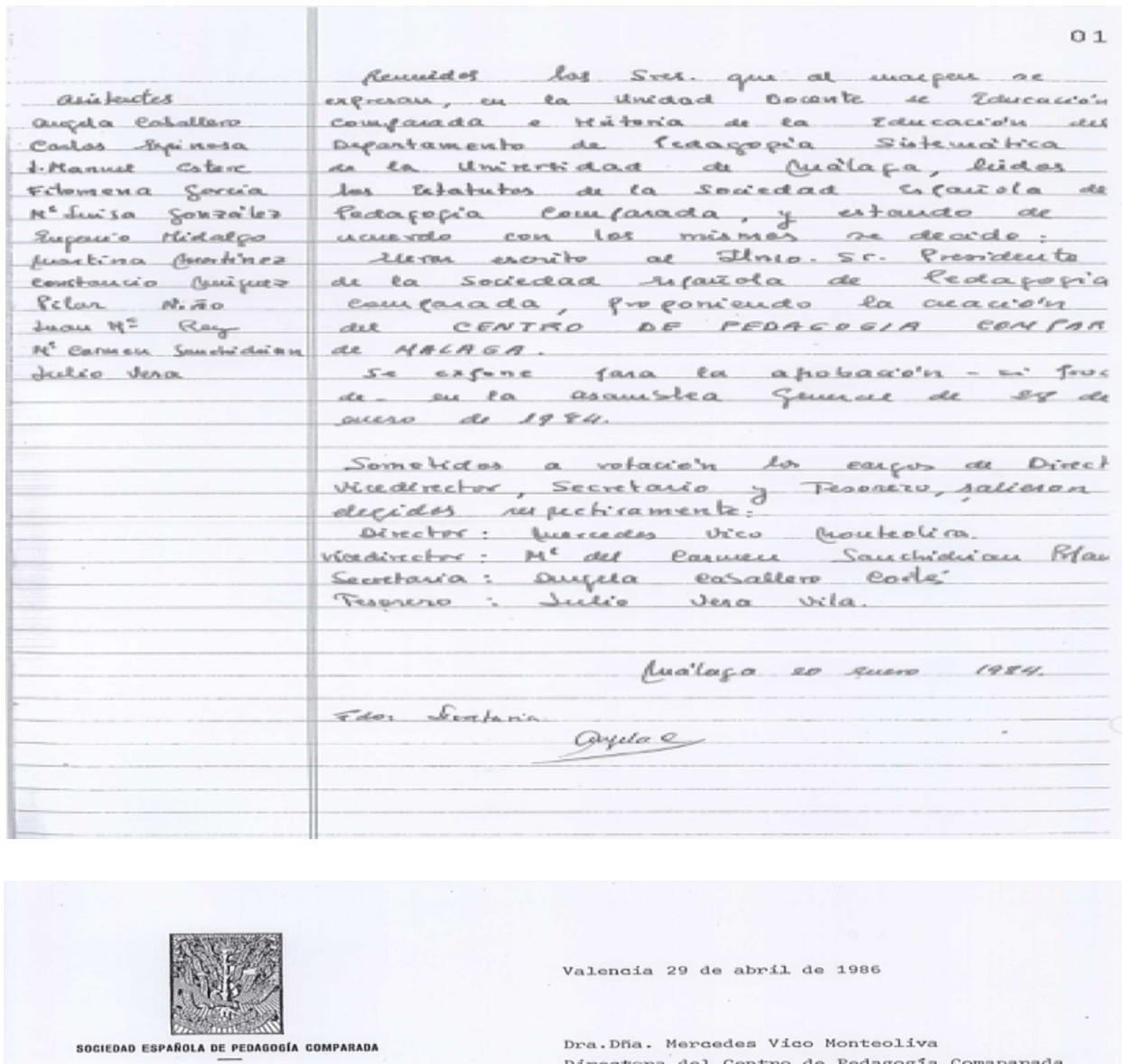

Dra.Dra. Mercedes Vico Monteoliva

Directora del Centro do Pedagogia Comaparade Universidad de Malaga

MALAGA

Eatimada amiga y companera, en relación a tu petición de namero

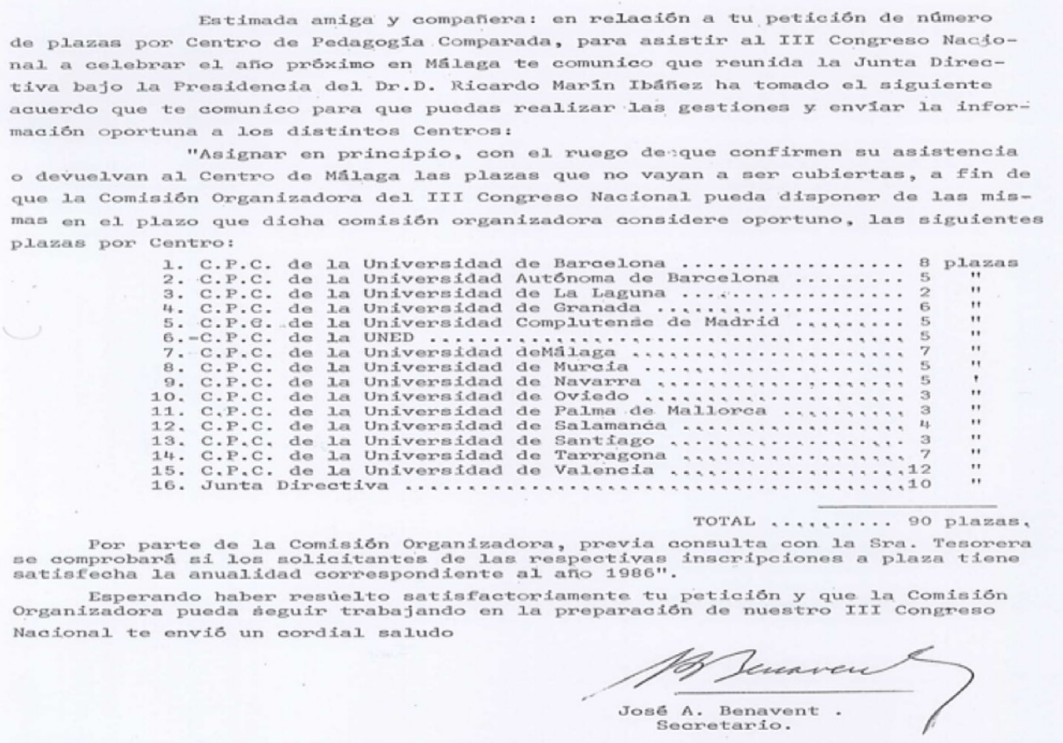

Y según el libro de actas, depositado en la Secretaria del Departamento, junto a toda la documentación generada por el Centro creado en 1984, se da cuenta de la última sesión celebrada en Málaga

\footnotetext{
${ }^{13}$ En el acta del día 18 de febrero de 1985, « se dialoga sobre la posibilidad de ofrecer Málaga como sede del III Congreso Nacional de Pedagogía Comparada». Se acepta por los presentes y se le comunica al Presidente de la Sociedad.
} 
el día 19 de mayo de 1994. En ella Mercedes Vico y Ángela Caballero, informan de la reunión anual de la Sociedad, celebrada en Madrid, y de cómo se contempla la asignatura de Educación Comparada en la titulación de Pedagogía. En el Boletín de la Sociedad Española de Pedagogía Comparada, número 9, año 1986, se informa del Titulo del mismo.» LA UNIVERSIDAD EN EL MUNDO DE HOY», de las fechas del III Congreso, del 26 al 29 de marzo de 1987, del programa, así como del plano de situación del centro de Formación de Ojén. Se celebrará en la Fundación HANNS SEIDEL de Marbella (Málaga). Es en el Boletín de la Sociedad Española de Pedagogía Comparada, número 10, donde se da una amplia información del III Congreso Nacional de la S.E.P.C. Presentación, mesas de trabajo y conclusiones...

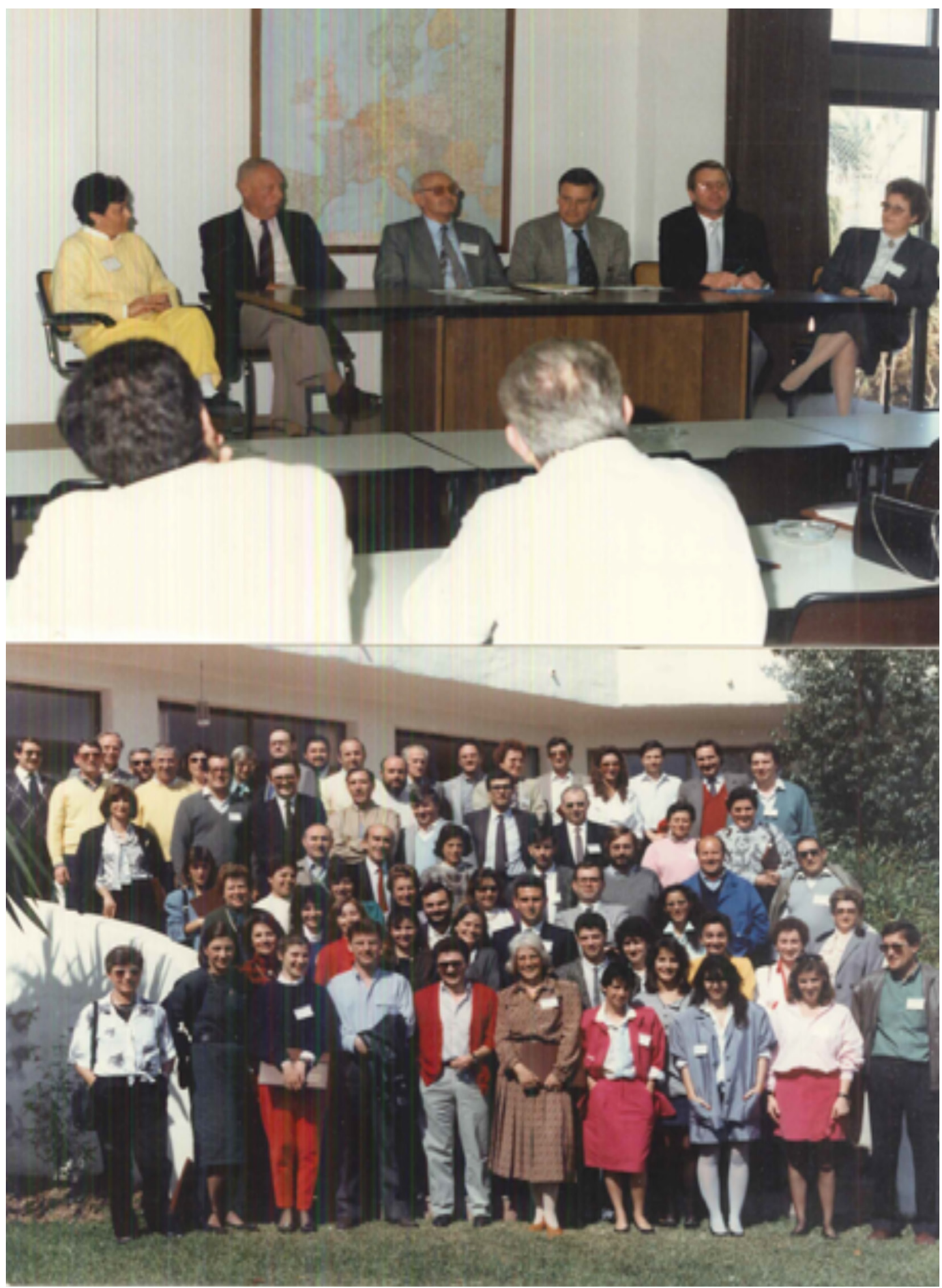

Fotografías del III Congreso Nacional de Educación Comparada . Fundación Hanns-Seidel. Marbella (Málaga) 1987. 
PLAN DE ESTUDIOS DE FILOSOFIA Y CIENCIAS DE LA EDUCACIÓN. Orden de 16 de noviembre de 1983 por la que se aprueba la modificación del Plan de Estudios de la División de Filosofía y Ciencias de la Educación de la Facultad de Filosofía y Letras de la Universidad de Málaga (B.O.E. 27 de enero de 1984). DIVISION DE FILOSOFIA Y CIENCIAS DE LA EDUCACIÓN. Sección de Ciencias de la Educación.

\begin{tabular}{|c|c|c|c|}
\hline & ASIGNATURAS & & ASIGNATURAS \\
\hline & Historia de la Filosofía & & \\
\hline & Introducción Emp. a las C. de la Educ. & & Historia de la Educación II \\
\hline \multirow[t]{6}{*}{$\begin{array}{l}\text { PRIMER } \\
\text { CURSO }\end{array}$} & Estadística aplicada a las C. Humanas I & $\begin{array}{l}\text { CUARTO } \\
\text { CURSO }\end{array}$ & Didáctica II \\
\hline & Fundamentos Biológicos de la Conducta & & $\begin{array}{l}\text { Organización y Legislación } \\
\qquad \text { Escolar }\end{array}$ \\
\hline & Psicología General & & Orientación Educativa I \\
\hline & & $\begin{array}{c}\text { Dos cursos } \\
\text { cuatrimestrales }\end{array}$ & $\begin{array}{c}\text { a elegir entre los que cada } \\
\text { año organice la Sección }\end{array}$ \\
\hline & Pedagogía General & & \\
\hline & Psicología Evolutiva & & Pedagogía Experimental \\
\hline \multirow[t]{5}{*}{$\begin{array}{l}\text { SEGUNDO } \\
\text { CURSO }\end{array}$} & Estadística aplicada a las C. Humanas & QUINTO CURSO & $\begin{array}{l}\text { Psicología de la } \\
\text { Educación }\end{array}$ \\
\hline & Sociología de la Educación & & Didáctica Especial \\
\hline & Didáctica I & & Educación Especial \\
\hline & Psicodiagnóstico & CURSOS & CUATRIMESTRALES \\
\hline & Psicopatología & $\begin{array}{l}\text { Educación } \\
\text { Comparada }\end{array}$ & $\begin{array}{c}\text { Historia de las Instituciones } \\
\text { docentes. en España }\end{array}$ \\
\hline \multirow[t]{3}{*}{$\begin{array}{l}\text { TERCER } \\
\text { CURSO }\end{array}$} & Psicometría & $\begin{array}{l}\text { Pedagogía } \\
\text { Diferencial }\end{array}$ & Orientación Educativa II \\
\hline & Filosofía de la Educación & $\begin{array}{c}\text { Diseños } \\
\text { Experimentales }\end{array}$ & Pedagogía Social \\
\hline & Historia de la Educación I & $\begin{array}{l}\text { Tecnología de la } \\
\text { Educación }\end{array}$ & Planificación Educativa \\
\hline
\end{tabular}

\section{CONCLUSIONES}

Con poco más de 45 años de vida, la Universidad de Málaga se ha convertido en una gran institución docente, conectada con la sociedad, siendo el punto de referencia en la investigación y en el campo tecnológico. La UMA, vive actualmente un gran proceso de trasformación, con la integración de todos sus centros, y los de próxima construcción, en una ciudad universitaria ubicada en el Campus de Teatinos.

La oferta del número de titulaciones, así como el número de alumnos es más numerosa, el aumento del número de profesores, el personal de administración y servicios...es una realidad. A todo ello hay que sumar la adaptación al Espacio Europeo de Educación Superior, un nuevo reto, cuyos resultados veremos dentro de una década.

En palabras del Rector: « Quiero agradeceros a todos, en nombre del equipo de gobierno y en el mío propio, vuestro trabajo, vuestra colaboración y vuestra confianza durante este año y emplazaros para seguir trabajando juntos en hacer que la Universidad de Málaga sea un modelo de institución pública al servicio de la sociedad y de la que todos nos podamos sentir orgullosos». 


\section{REFERENCIAS BIBLIOGRÁFICAS}

XXV (1998). Años de la Universidad de Málaga.

EI PENDULO. (1993). Revista de Industria Ciencia y Tecnología. «La Universidad de Málaga. 20 años de Historia». Monográfico, número 5, Málaga. U.M.A. (2000-20010). Estadísticas de alumnos . Servicio Central de Informática.

Guía de la Universidad de Málaga. http://www.uma.es.

Boletín de la Sociedad Española de Educación Comparada.: http://www.sc.ehu.es/sfwseec/boletin.htm. Revista Española de Educación Comparada (REEC): http://www.uned.es/reec/espanol.html y http://www.uned.es/reec/espanol.html. 